\title{
Susceptibilidad antimicrobiana y bases genéticas de la resistencia de cepas de Enterococcus causantes de infecciones en Cuba
}

\author{
Dianelys Quiñones Pérez, ${ }^{1}$ Miriam Abreu Capote, ${ }^{1}$ Deisy Marrero, ${ }^{2}$ \\ Ana Bertha Alvarez, ${ }^{3}$ Cecilia Ortiz, ${ }^{4}$ Francisco Salomé, ${ }^{5}$ \\ Alina Llop 6 y Rosa del Campo ${ }^{7}$
}

Forma de citar

Quiñones Pérez D, Abreu Capote M, Marrero D, Alvarez AB, Ortiz C, Salomé F, et al. Susceptibilidad antimicrobiana y bases genéticas de la resistencia de cepas de Enterococcus causantes de infecciones en Cuba. Rev Panam Salud Publica. 2011;30(6):549-54.

ABSTRACT Objetivo. Identificar las especies de Enterococcus causantes de infecciones en hospitales cubanos, su susceptibilidad a los antimicrobianos y sus mecanismos de resistencia.

Métodos. Se estudiaron 687 aislamientos de Enterococcus procedentes de 30 hospitales cubanos de nueve provincias del país durante el período de 2000 a 2009. La identificación de las especies se realizó mediante el método convencional y sistema automatizado API ${ }^{\circledR}$ La concentración inhibitoria minima se determinó para 13 antimicrobianos según las recomendaciones del Instituto de Estándares Clínicos y de Laboratorio. Se determinaron los genes de resistencia a aminoglucósidos, eritromicina, tetraciclina y glucopéptidos mediante reacción en cadena de la polimerasa. La presencia de betalactamasa se determinó por el método de la cefalosporina cromógena.

Resultados. Las especies más prevalentes fueron Enterococcus faecalis $(82,9 \%)$ y Enterococcus faecium $(12,2 \%)$. La resistencia a los glucopéptidos $(1,0 \%)$ estuvo mediada por los genes vanA y vanB y las cepas resistentes a ampicilina (6\%) no produjeron betalactamasas. Se observó un alto porcentaje de resistencia a los aminoglucósidos: gentamicina $(31,0 \%)$ y estreptomicina y amikacina $(29,1 \%)$ mediada por los genes aac $\left(6^{\prime}\right) \mathrm{Ie}-\mathrm{aph}\left(2^{\prime \prime}\right) \mathrm{Ia}$, aph(3')-IIIa, ant(6)Ia, ant(3")(9). Hubo correlación entre la resistencia a tetraciclina $(56,0 \%)$ y la presencia de los genes tet $(M)(75,1 \%)$ y tet $(L)(7,0 \%)$, mientras que la resistencia a eritromicina $(34,1 \%)$ obedeció al gen erm(B) $(70,9 \%)$.

Conclusiones. La resistencia a vancomicina es infrecuente en Cuba, a diferencia del alto nivel de resistencia a los aminoglucósidos, que sugiere posibles fracasos terapéuticos. El laboratorio de microbiología constituye un pilar fundamental de la vigilancia de las infecciones por cepas de Enterococcus y el monitoreo continuo de su susceptibilidad a los antimicrobianos, dado el incremento de la resistencia de ese microorganismo en el tiempo.

Palabras clave Enterococcus; farmacorresistencia bacteriana; Cuba.

1 Instituto de Medicina Tropical "Pedro Kouri", Departamento de Bacteriología-Micología, La Habana, Cuba. Enviar correspondencia a: Dianelys Quiñones Pérez, diany.quinones@infomed.sld.cu

2 Hospital Pediátrico "Octavio de La Concepción y la Pedraja", Servicio de Microbiología, Holguín, Cuba.
Hospital "Eusebio Hernández", Servicio de Microbiología, La Habana, Cuba.

4 Hospital "Ramón Gonzalez Coro", Servicio de Microbiología, La Habana, Cuba.

5 Centro Provincial de Higiene y Epidemiología, Cienfuegos, Cuba.
6 Instituto de Medicina Tropical "Pedro Kouri", Departamento de Microbiología, La Habana, Cuba.

Hospital Universitario "Ramón y Cajal", Servicio de Microbiología, Madrid, España. 
La bacteria del género Enterococcus constituye uno de los principales agentes patógenos nosocomiales en todo el mundo. Las especies E. faecalis y E. faecium son las más comunes, y causan infecciones del tracto urinario, heridas quirúrgicas y torrente sanguíneo, además de endocarditis y sepsis neonatal, entre otras (1-3). Este microorganismo tiene entre sus características principales una resistencia intrínseca a los antimicrobianos y amplia resistencia adquirida, que limitan considerablemente las opciones terapéuticas, por lo cual el control de la infección es problemático (4).

La vigilancia del género Enterococcus es imprescindible en todos los países porque dicha bacteria genera brotes de infección intrahospitalaria por cepas resistentes; presenta dificultades para detectar en el laboratorio los diferentes fenotipos de resistencia; ocasiona problemas terapéuticos, y tienen el potencial de diseminar genes de resistencia a otros microorganismos grampositivos. Por tales motivos, el personal del Laboratorio Nacional de Referencia de Microbiología del Instituto de Medicina Tropical "Pedro Kouri" (LNRM/IPK) está continuamente mejorando la vigilancia microbiológica de las cepas de Enterococcus causantes de infecciones en Cuba. En este trabajo se exponen los resultados de la vigilancia durante el período de 2000 a 2009, con el propósito de dar a conocer las especies circulantes, su susceptibilidad a los antimicrobianos y las bases genéticas de la resistencia.

\section{MÉTODOS}

Se realizó un estudio descriptivo de 687 aislamientos pertenecientes al género Enterococcus recibidos en el LNRM/ IPK entre el 3 de enero de 2000 y el 31 de diciembre de 2009. Los aislamientos procedieron de 30 hospitales de nueve provincias del país (Ciudad de La Habana, Cienfuegos, Sancti Spíritu, Ciego de Ávila, Camagüey, Holguín, Las Tunas, Santiago de Cuba y Guantánamo). Del total de aislamientos, $88 \%$ procedieron de pacientes hospitalizados y $12 \%$, de la comunidad. Estos últimos no registraron ingreso hospitalario durante el año precedente al de la obtención de la muestra. En el cuadro 1 se muestra la distribución de los aislamientos estudiados según el tipo de muestra analizado.

La identificación de género y especie se realizó por métodos convencionales

\section{CUADRO 1. Distribución de los 687 aislamientos estudiados según la procedencia de la muestra}

\begin{tabular}{llcc}
\hline \multirow{2}{*}{ Tipo de aislamiento } & No. de & \\
\hline Invasivo & Procedencia clínica & aislamientos & Porcentaje \\
& Sangre & 145 & 21,10 \\
& Líquido peritoneal & 23 & 3,34 \\
& Líquido cefalorraquídeo & 15 & 2,18 \\
& Líquido amniótico & 3 & 0,44 \\
Total & Tejidos & 4 & 0,58 \\
& & 190 & 27,60 \\
& Orina & 114 & 16,60 \\
& Herida quirúrgica & 90 & 13,10 \\
No invasivo & Punta de catéter & 48 & 6,99 \\
& Vagina & 31 & 4,51 \\
& Oído & 23 & 3,35 \\
& Piel & 19 & 2,78 \\
Total & Uretra & 13 & 1,89 \\
Portadores fecales & Secreción endotraqueal & 11 & 1,60 \\
Total de cepas & Otro & 75 & 10,90 \\
& & 424 & 61,70 \\
& Heces & 73 & 10,60 \\
& & 687 & 100,00 \\
\hline
\end{tabular}

Fuente: Laboratorio Nacional de Referencia de Microbiología del Instituto de Medicina Tropical "Pedro Kourí".

Otros: vulva (19), faringe (14), esputo (11), loquios (9), secreción de gastrostomía (5), sonda de levín (4), drenaje hepático (4), prepucio (4), fístula perianal (3), canal radicular dental (2).

definidos por Facklam y Collins (5). Se emplearon el sistema Mini Api ${ }^{\circledR}$ y las galerías Rapid ID 32 STREP ${ }^{\circledR}$ (BioMérieux, Francia) para confirmar la identificación de especie. Se utilizaron como control las siguientes cepas: E. faecalis ATCC 29212, E. faecium ATCC 6056 y Enterococcus casseliflavus ATCC 25788. Como control negativo para los carbohidratos se utilizó el caldo básico de rojo fenol (Difco, Alemania) sin la adición de azúcares.

La susceptibilidad a los antimicrobianos se determinó mediante la concentración inhibitoria mínima (CIM) para vancomicina, teicoplanina, ampicilina, gentamicina, estreptomicina, amikacina, nitrofurantoína, eritromicina, tetraciclina, ciprofloxacina, levofloxacina, norfloxacina y moxifloxacina, según las recomendaciones del Instituto de Estándares de Laboratorio Clínico (CLSI, por su sigla en inglés) (6); se utilizó la cepa E. faecalis ATCC 29212 como control. Para las cepas resistentes a la ampicilina se determinó la producción de betalactamasa por el método de la cefalosporina cromógena (Nitrocefin, Oxoid, Basingstoke, Inglaterra), según las instrucciones del fabricante. Se usaron como controles las cepas Escherichia coli ATCC 35218 (control positivo) y Neisseria gonorrhoeae ATCC 29216 (control negativo).
La detección de genes de resistencia a los antimicrobianos se realizó por reacción en cadena de polimerasa convencional.

Se investigó la presencia de los genes de resistencia a aminoglucósidos [aac $\left(6^{\prime}\right)$ Ie-aph(2")Ia, aph(3')-IIIa, ant(6)Ia, ant(3") (9), $\operatorname{aph}\left(2^{\prime \prime}\right)-i d$ y aph (2")-ic]; glucopéptidos [van(A) y $\operatorname{van}(\mathrm{B})]$; macrólidos [erm(B), $\operatorname{erm}(\mathrm{A}), \operatorname{erm}(\mathrm{C})$ y mef(A)] y tetraciclina $[\operatorname{tet}(\mathrm{M}), \operatorname{tet}(\mathrm{L})$, tet $(\mathrm{K}), \operatorname{tet}(\mathrm{O}), \operatorname{tet}(\mathrm{S}), \operatorname{tet}(\mathrm{T})$ $\mathrm{y}$ tet $(\mathrm{U})]$ en los aislamientos resistentes. Se usaron cebadores específicos según protocolos estandarizados previamente (7-12) y cepas controles positivas. Como control negativo se utilizó agua destilada estéril.

\section{Análisis estadístico}

Los resultados obtenidos se procesaron mediante el programa Microsoft Office Excel 2003. Se utilizaron medidas de estadística descriptiva, como la frecuencia (\%), para el análisis y la presentación de los resultados.

\section{RESULTADOS}

La especie E. faecalis fue la más prevalente $(82,9 \%)$, seguida por las cepas de E. faecium $(12,2 \%)$, E. gallinarum $(1,4 \%)$, E. avium $(1,3 \%)$, E. casseliflavus $(0,7 \%), E$. hirae $(0,6 \%)$, E. durans $(0,4 \%)$ y E. raffinos- 
sus $(0,4 \%)$. En la figura 1 se muestran los antimicrobianos de las cepas de Enterococcus caracterizadas.

El 31,0\% de las cepas presentó alto nivel de resistencia a gentamicina, mientras que la resistencia a estreptomicina y amikacina fue de $29,1 \%$. Los altos niveles de resistencia a los aminoglucósidos detectados obedecieron principalmente a un mecanismo enzimático. Todos los aislamientos con alto nivel de resistencia a gentamicina portaban el gen aac $\left(6^{\prime}\right)$ Ie-aph(2")Ia (figura 2) que codifica la enzima bifuncional AAC(6')-APH(2"). porcentajes de resistencia a los diferentes

En los aislamientos con alto nivel de resistencia a amikacina, 35,0\% presentaban la misma enzima bifuncional y 33,0\% el gen $\operatorname{aph}\left(3^{\prime}\right)-I I I a ; 32,0 \%$ presentaron ambos mecanismos. En los aislamientos con alto nivel de resistencia a estreptomicina predominó el gen ant(6)-Ia $(49,0 \%)$, seguido por el gen ant( $\left.3^{\prime \prime}\right)(9)(25,0 \%)$ y en $10,0 \%$ se observaron ambos mecanismos. En $15,0 \%$ de los aislamientos no se identificó ningún mecanismo enzimático como causa de la resistencia de alto nivel a estreptomicina.

De los aislamientos con alto nivel de resistencia a amikacina, 67,0\% tenían

FIGURA 1. Resistencia a diferentes antimicrobianos (\%) de 687 cepas de Enterococcus estudiadas, Cuba, 2000 a 2009

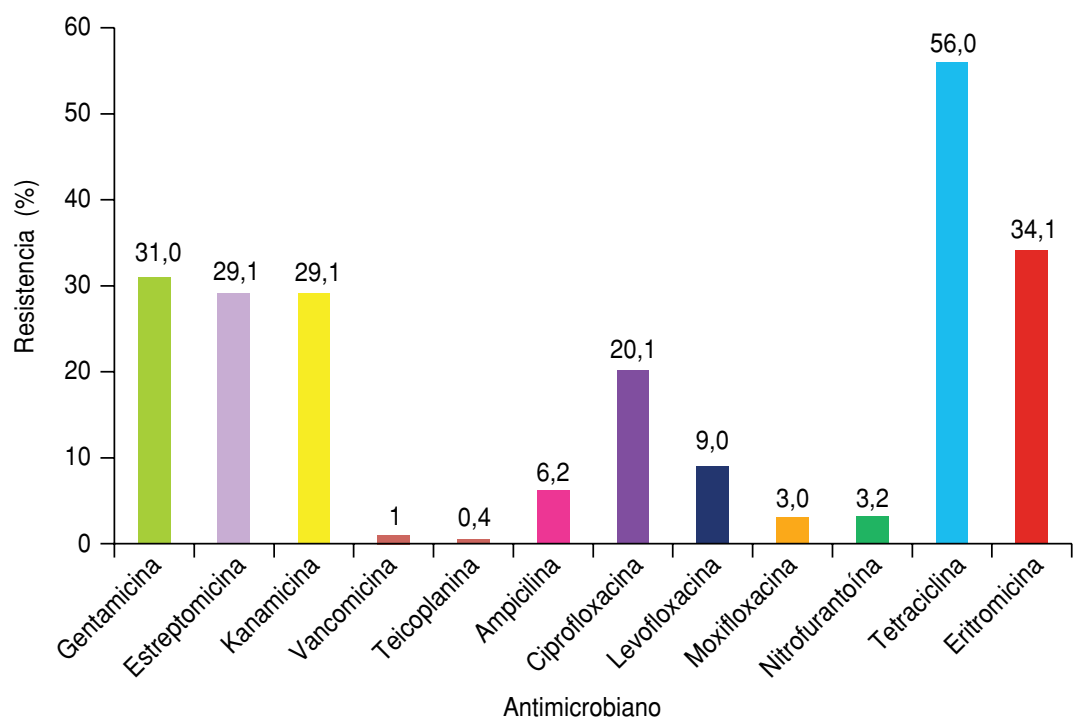

FIGURA 2. Electroforesis en gel de agarosa de los productos de la amplificación de los genes de las enzimas modificadoras de aminoglucósidos

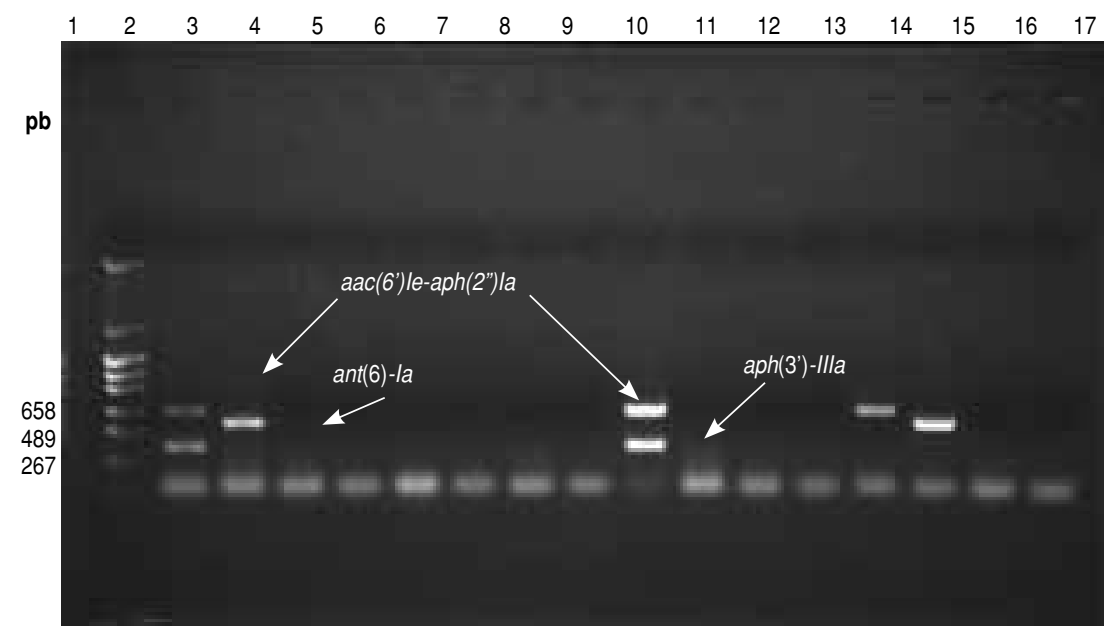

Línea 1: marcador de peso molecular; línea 2 control positivo: aac(6’)le-aph(2") la y aph(3')-Illa; línea 3 control positivo: ant(6)-la; líneas 10 y 14: producto amplificado aac(6') le-aph(2") la de 675 pb; línea 10: producto amplificado aph(3')-IIla de 354 pb; línea 15: producto amplificado ant(6)-la de 548 pb; líneas: 16 y 17 controles negativos; líneas 4-9 y 11-13: resultados negativos. más de un tipo de gen codificante de enzimas modificadoras de aminoglucósidos. Las combinaciones más frecuentes fueron: $\operatorname{aac}\left(6^{\prime}\right)-\operatorname{aph}\left(2^{\prime \prime}\right)+\operatorname{aph}\left(3^{\prime}\right)-I I I a+$ ant(6)-Ia: 19,0\%; aac (6')-aph(2") + ant(6)Ia: $17,0 \% ; \operatorname{aac}\left(6^{\prime}\right)-\operatorname{aph}\left(2^{\prime \prime}\right)+\operatorname{aph}\left(3^{\prime}\right)-I I I a+$ ant (3")(9): 2,0\%; aac (6')-aph( $\left.2^{\prime \prime}\right)+\operatorname{aph}\left(3^{\prime}\right)-$ IIIa: $10,0 \%$.

Por otra parte, se observó un bajo porcentaje de resistencia a ampicilina $(6,2 \%)$, y ninguna de las cepas produjo betalactamasa. Asimismo, se detectó una baja proporción de resistencia a los glucopéptidos ( $1 \%$ a vancomicina y $0,4 \%$ a teicoplanina), que en $50 \%$ de las cepas estuvo mediada por los genes van $A$ (figura 3) en los aislamientos con una $\mathrm{CIM} \geq 256 \mu \mathrm{g} / \mathrm{ml}$ para vancomicina $\mathrm{y}$ $\geq 32 \mu \mathrm{g} / \mathrm{ml}$ para teicoplanina, y por el gen $\operatorname{van} \mathrm{B}(50,0 \%)$ en los aislamientos con resistencia a vancomicina (CIM $\geq 256$ $\mu \mathrm{g} / \mathrm{ml})$. La nitrofurantoína fue activa contra la mayoría de las cepas estudiadas. Con respecto a las fluoroquinolonas, se observaron valores de resistencia que oscilaron entre 3,0\% para moxifloxacina y $20,1 \%$, para ciprofloxacina (figura 1 ).

Se detectó resistencia elevada a la tetraciclina, que obedeció a los dos mecanismos descritos con más frecuencia: el gen tet(M) en 75,1\% y el gen tet(L) en $7,0 \%$ de las cepas (figura 4). También se observó un porcentaje elevado de resistencia a la eritromicina con la detección del gen erm(B) en 70,9\% de los aislamientos (figura 5). No se detectó ningún gen causante de resistencia en $29,1 \%$ de los aislamientos.

\section{DISCUSIÓN}

La prevalencia de especies enterocócicas entre 2000 y 2009 en Cuba fue similar

FIGURA 3. Electroforesis en gel de agarosa del producto de amplificación del gen vanA

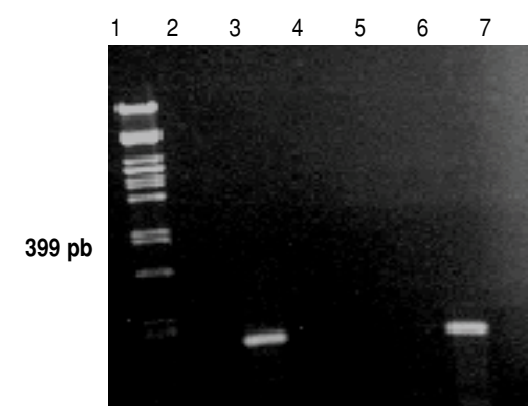

Línea 1: marcador de peso molecular; línea 2: control negativo; línea 3: vanA control positivo; líneas 4-6: reacciones negativas; línea 7: producto amplificado de 399 pb del gen vanA. 
FIGURA 4. Electroforesis en gel de agarosa del producto de amplificación de los genes tet(M) y $\operatorname{tet}(\mathrm{L})$

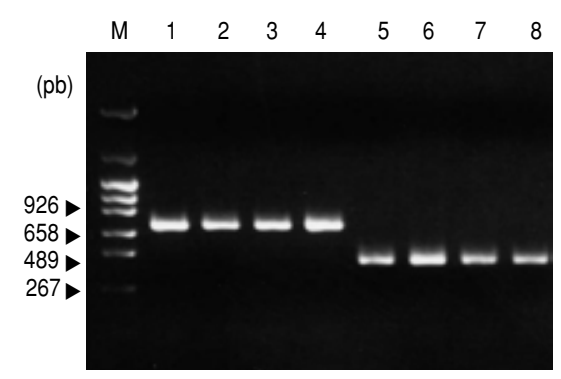

Línea 1: marcador de peso molecular; líneas 2-5: producto amplificado de tet $(\mathrm{L})$ de $740 \mathrm{pb}$; líneas 6-9: producto amplificado de tet(M) de $435 \mathrm{pb}$.

FIGURA 5. Electroforesis en gel de agarosa del producto de amplificación del gen erm(B)

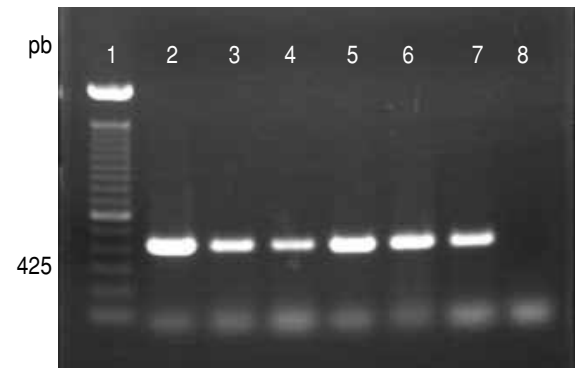

Línea 1: marcador de peso molecular; línea 2: control positivo; líneas 3-7: amplificado erm(B) de $425 \mathrm{pb}$; línea 8: control negativo.

a la informada internacionalmente, pues las especies $E$. faecalis y E. faecium son las de mayor importancia clínica, mientras que las especies E. gallinarum, E. raffinossus, E. casseliflavus, E. avium, E. durans y E. hirae constituyen menos de $10 \%$ de los aislamientos enterocócicos $(4,13)$. El predominio de las cepas de E. faecalis puede obedecer a su mayor patogenicidad (14) y a una mejor adaptación al huésped humano. No obstante, actualmente, se observa un aumento de la prevalencia de cepas de E. faecium relacionado con un incremento de su resistencia a los antimicrobianos y la adquisición de islas de patogenicidad $(3,15)$.

Los aislamientos con alto nivel de resistencia a amikacina detectados son similares a los informados en trabajos internacionales (16-18); la resistencia guardó relación con las enzimas modificadoras de aminoglucósidos, mecanismo principal de resistencia detectado en las cepas cubanas. El alto nivel de resistencia a gentamicina está relacionado con la enzima bifuncional $\mathrm{AAC}\left(6^{\prime}\right)-\mathrm{APH}\left(2^{*}\right)$, que es la mayor causa de fracaso del tratamiento con aminoglucósidos, ya que su factor genético determinante es el más difundido entre las cepas con alto nivel de resistencia a gentamicina (16).

La alta resistencia a amikacina mediada por la enzima bifuncional y el gen aph(3')-IIIa es similar a la descrita por Emaneini y colaboradores, quienes encontraron el gen aph(3')-IIIa en 37\% de sus aislamientos y mostraron la coexistencia $\operatorname{aph}\left(3^{\prime}\right)-I I I+\operatorname{aac}\left(6^{\prime}\right) I e-a p h\left(2^{\prime \prime}\right) I a$ en $28 \%$ y $49 \%$ de los aislamientos de E. faecalis y E. faecium, respectivamente (17). Los elevados porcentajes de detección del gen ant(6)-Ia $(49,0 \%)$ son similares a los descritos por Watanabe y colaboradores en Japón, donde se notificó que hasta $56,5 \%$ de las cepas de E. faecium eran portadoras de ese gen (19). En $15,0 \%$ de los aislamientos con alto nivel de resistencia a estreptomicina en los que no se detectó un mecanismo enzimático, la resistencia puede deberse a una posible mutación ribosómica (18).

La ampicilina es un antibiótico de primera línea para el tratamiento de la infección enterocócica (20). La resistencia detectada en relación con este antimicrobiano no constituye hoy un problema terapéutico, sin embargo, es importante mantener la vigilancia debido a la diseminación mundial de la cepa E. faecium CC17 resistente a la ampicilina (15). Dicha resistencia podría obedecer a una baja afinidad de las proteínas fijadoras de penicilinas tipo 5 , ya que no se detectó la producción de betalactamasa, mecanismo enzimático infrecuente en las cepas de Enterococcus (0,1\%) (21).

La baja resistencia a glucopéptidos detectada puede obedecer al uso controlado y racional de la vancomicina y la teicoplanina que se realiza en los centros sanitarios cubanos. En América Central y América del Sur también se observan bajas tasas de prevalencia de enterococos resistentes a vancomicina, aunque en el Brasil se notificó un incremento de 6,9\% a 31,1\% entre 2003 y 2008, respectivamente $(22,23)$. En los Estados Unidos, la resistencia a glucopéptidos constituye un problema grave y se observa en $80 \%$ de los aislamientos de E. faecium (22). La detección de los genes $\operatorname{van} \mathrm{A}$ y $\operatorname{van} \mathrm{B}$ en cepas de Enterococcus cubanas es comparable a la informada por Fica y colaboradores en Chile, quienes también detectan ambos genes (24). En Cuba se notificó un aislamiento de Enterococcus resistente a vancomicina portador del gen van $\mathrm{A}$, por lo que este genotipo, aunque raro todavía en el país, podría verse con mayor frecuencia en el futuro (25).

Con respecto a las fluoroquinolonas, los porcentajes de resistencia encontrados coinciden con los descritos en otros países, y reflejan una mejor actividad de la moxifloxacina in vitro frente a cepas de Enterococcus (26).

Dado que la resistencia de los enterococos a nitrofurantoína es baja, ese fármaco es una buena opción terapéutica para la infección urinaria por ese agente patógeno. En general, la resistencia a nitrofurantoína no es alta en el ámbito internacional, e incluso muestra buena actividad in vitro frente a las cepas de enterococos resistentes a vancomicina (27).

La resistencia elevada a tetraciclina y eritromicina detectada es coherente con la amplia diseminación de esa resistencia entre cepas del género Enterococcus (28). Estos resultados pueden ser consecuencia del alto consumo de esos fármacos (29), que ejerce presión selectiva positiva sobre las cepas resistentes a tetraciclina y eritromicina. Además, los determinantes que codifican estas resistencias están localizados en plásmidos y transposones y se diseminan entre las distintas cepas (30). Los genes que mediaron la resistencia a tetraciclina fueron tet(M), que es el más frecuente de los del género Enterococcus (31) y tet (L), con menor prevalencia (entre $10 \%$ y $42 \%$ ) $(32,33)$. Los resultados del presente trabajo ratifican este hecho.

La resistencia a eritromicina obedeció, principalmente, a la presencia del gen $\operatorname{erm}(\mathrm{B})$, por lo que se relaciona con la metilación del ARNr 235 debido a la acción de las metilasas Erm(B). Con base en estos resultados y en datos publicados previamente, se demuestra que este mecanismo es universal y está muy diseminado entre las cepas de Enterococcus (28). En 29,1\% de los aislamientos no se detectó ningún gen de resistencia, por lo que esa pudo haber obedecido a una mutación cromosómica, que es otro de los mecanismos descritos (34).

Como limitaciones del estudio se debe señalar que, debido a su diseño, sin previa selección de cepas que fuesen representativas del país, los resultados no pueden extrapolarse a otras provincias no incluidas en el trabajo; tampoco son 
evidencia de la situación real de la resistencia de las cepas de Enterococcus en el ámbito nacional. Otra limitante es que, a pesar de que se describe el porcentaje de aislamientos de origen intrahospitalario y de la comunidad, no se realizó un análisis del tipo de infección ni del comportamiento de la resistencia a los antimicrobianos según el servicio donde se obtuvo el aislamiento, por lo que no se muestran las diferencias que podría haber entre las cepas provenientes de ambos lugares, ni en el comportamiento de la resistencia a los antimicrobianos según estos hábitat.

\section{Conclusiones}

Las infecciones por cepas de Enterococcus faecalis y E. faecium constituyeron las de mayor importancia clínica en el período de estudio. La resistencia a los antimicrobianos detectada es equivalente a la situación internacional y constituye una advertencia sobre fracasos terapéuticos potenciales debido al tratamiento con aminoglucósidos. La ampicilina, los glucopéptidos y la nitrofurantoína mostraron muy buena actividad in vitro. No obstante, la detección de los genes van $\mathrm{A}$ y van $\mathrm{B}$ demandan una vigilancia continua y estricta de las cepas de Enterococcus resistentes a vancomicina en el país. Se recomienda fortalecer la vigilancia microbiológica de esta bacteria con cepas representativas del país, así como llevar a cabo estudios de epidemiología molecular para la detección de clones emergentes multirresistentes.

Agradecimientos. A todo el personal de los laboratorios de microbiología de la red nacional cubana y de los centros provinciales de higiene y epidemiología que contribuyen con la vigilancia de los aislamientos de Enterococcus en Cuba.

\section{REFERENCIAS}

1. Sood S, Malthotra M, Das B, Kapil A. Enterococcal infection and antimcirobial resistance. Indian J Med Res. 2008;128:111-21.

2. Chen A, Zerbos M. Enterococcus: antimicrobial resistance in enterococci, epidemiology, treatment, and control. En: Mayera DL. editor. Antimicrobial Drug Resistance. New York: Humana Press; 2009. Pp. 715-33.

3. Bourdon N, Fines-Guyon M, Thiolet JM, Maugat S, Caignard B, Leclerq $\mathrm{R}$ et al. Changing trends in vancomycin-resistant enterococci in French hospitals, 2001-08. J Antimicrob Chemother. 2011;66:713-21.

4. Upadhyaya PM, Umapathy BL, Ravikumar KL. Comparative study for the presence of enterococcal virulence factors gelatinase, hemolysin and biofilm among clinical and commensal isolates of Enterococcus Faecalis. J Lab Physicians. 2010;2(2):100-4.

5. Facklam RR, Collins MD. Identification of Enterococcus species isolated from human infections by a conventional test scheme. J Clin Microb. 1989;27:731-4.

6. Clinical and Laboratory Standards Institute. Performance standards for antimicrobial susceptibility testing; 17th informational supplement. CLSI document M100-S17. Table 2D Enterococcus spp. M7-A7- MIC Testing. Wayne (PA): Clinical and Laboratory Standards Institute; 2007.

7. Van de Klundert JAM, Vliegenthart JS. PCR detection of genes coding for aminglycosidemodifying enzymes. En: Persing DH, Smith TF, Tenover FC, White TJ (editores). Diagnostic molecular microbiology principles and applications. Washington, DC: Press; 1993. Pp. 547-55.

8. Swenson JM, Ferraro MJ, Sham DF. Multilaboratory evaluation of screening methods for detection of high-level aminoglycoside resistance in enterococci. J Clin Microbiol. 1995;33:3008-18.

9. VakulenkoSB, DonabedianSM, Voskresenskiy AM. Multiplex PCR for detection of aminoglycoside resistance genes in enterococci. Antimicrob Agents Chemother. 2003;47:1423-26.

10. Clark NM, Hershberger E, Zervosc MJ, Lynch JP. Antimicrobial resistance among gram- positive organisms in the intensive care unit. Curr Opin Crit Care. 2003;9(5):403-12.

11. Sutcliffe J, Grebe T, Tait-Kamradt A, Wondrack L. Detection of erythromycinresistant determinants by PCR. Antimicrob Agents Chemother. 1996;40:2562-66.

12. Nishimoto $Y$, Kobayashi N, Alam M, Ishino M, Uehara N, Watanabe N. Analysis of the prevalence of tetracycline resistance genes in clinical isolates of Enterococcus faecalis and Enterococcus faecium in a Japanese hospital. Microb Drug Resist. 2005;11:146-53.

13. Diarra M, Rempel H, Champagne J, Masson L, Pritchard J, Topp E. Distribution of antimicrobial resistance and virulence genes in Enterococcus spp. and characterization of isolates from broiler chickens. Appl Environm Microbiol. 2010;76(24):8033-43.

14. Rahangdale VA, Agrawal G, Jalgaonkar SV. Study of antimicrobial resistance in enterococci. Indian J Med Microbiol. 2008;26:285-7.

15. Conceição N, De Oliveira C, Silva P, Godoi B, Ávila M, Gonçalves A. Trends in antimicrobial resistance among clinical isolates of enterococci in a Brazilian tertiary hospital: a 4-year study. Rev Soc Bras Med Trop. 2011;44(2):177-81.

16. Gajan E, Akbarzadeh P, Harasi B, Moosavi Z. Evaluation of genetic pattern of gentamicinresistant enterococci isolated from clinical samples. J Dent Res Dent Clin Dent Prospects. 2011;5(1):23-6.

17. Emaneini M, Aligholi M, Aminshahi M. Characterization of glycopeptides, aminoglycosides and macrolide resistance among E. faecalis and E. faecium isolates from a hospital in Teheran. Polish J of Microbiol. 2008;57(2):173-8.

18. Watanabe S, Kobayashi N, Quiñones D, Nagashima S, Uehara N, Watanabe N. Genetic Diversity of enterococci harboring the highlevel gentamicin resistance gene aac(6 0)-Ieaph(2 00)-Ia or aph(200)-Ie in a Japanese hospital. Microb Drug Resist. 2009;15(3):185-94.

19. Kobayashi N, Alam M. Prevalence and genetic diversity of aminoglycoside modifying enzymes in enterococci. En: Kobayashi N, Pandalai SG, editores. Drug resistance of enterococci: epidemiology and molecular mechanism. Trivandrum: Research Signpost; 2006. Pp. 209-27.

20. Yousef Khan F, Sitina S. Elshafi. Enterococcus gallinarum meningitis: a case report and literature review. Infect Dev Ctries. 2011;5(3): 231-4.

21. Rafii F. Betalactam resistance in enterococci. En: Kobayashi N, Pandalai SG, editores. Drug resistance of enterococci: epidemiology and molecular mechanism. Trivandrum: Research Signpost; 2006. Pp. 101-15.

22. Panesso D, Reyes J, Rincón S, Díaz L, Galloway-Peña J, Zurita J, et al. Molecular epidemiology of vancomycin-resistant Enterococcus faecium: a prospective, multicenter study in South American hospitals. J Clin Microbiol. 2010;48:1562-9.

23. Sader HS, Moet GJ, Jones RN. Antimicrobial resistance among Gram-positive bacteria isolated in Latin American hospitals. J Chemother. 2009;21(6):611-20.

24. Fica A, Jemenao MI, Bilbao P, Ruiz G, Sakurada A, Pérez de Arce E, et al. Emergency of vancomycin-resistant Enterococcus infections in a teaching hospital in Chile. Rev Chilena Infectol. 2007;24(6):462-71.

25. Espino M, Couto MJ, Rojas N, Fiol N, Torriente M. Análisis de episodios de sepsis en una unidad de cuidados intensivos neonatal. Rev Panam Infectol. 2005;7(2):22-8.

26. Hidalgo M, Reyes J, Cárdenas AM, Díaz L, Rincón S, Vanegas N, et al. Perfiles de resistencia a fluoroquinolonas en aislamientos clínicos de cocos gram-positivos provenientes de hospitales colombianos, 1994-2004. Biomédica. 2008;28:284-94.

27. Heintz BH, Halilovic J, Christensen CL. Vancomycin-resistant enterococcal urinary tract infections. Pharmacotherapy. 2010; 30(11):1136-49.

28. Zou L-K, Wang H-N, Zeng B, Li J-N, Li $\mathrm{X}-\mathrm{T}$, Zhang A-Y, et al. Erythromycin resistance and virulence genes in Enterococcus faecalis from swine in China. New Microbiologica. 2011;34:73-80.

29. Lara MC, Cires M, García AJ. Consumo de antimicrobianos en APS. Rev Cubana Med Gen Integr. 2003;19(4):1-2. 
30. Alebouyeh $\mathrm{M}$, Amirmozafari $\mathrm{N}$, Forohesh $\mathrm{H}$. Evaluation of virulenece factors and plasmad-realted transmissibility among different isolates of enterococci. Iranian Biomedical J. 2005;9(2):51-5.

31. Mitsou1 E, Kirtzalidou1 E, Pramateftaki P, Kyriacou1 A. Antibiotic resistance in faecal microbiota of Greek healthy infants. Benef Microbes. 2010;1(3):297-306.

32. Aarestrup FM, Agerso A, Gerner-Smidt P, Madsen M, Jensen LB. Comparison of antimicrobial resistance phenotypes and resistance genes in Enterococcus faecalis and Enterococcus faecium from humans in the community, broilers, and pigs in Denmark. Diagn Microbiol Infect Dis. 2000;37:127-37.

33. Huys G, D'Haene K, Collard JM, Swings J. Prevalence and molecular characterization of tetracycline resistance in Enterococcus isolates from food. Appl Environ Microbiol. 2004;70:1555-62.

34. Del Campo R, Ruiz-Garbajosa P, Sánchez Moreno P, Baquero F, Torres C, Cantón R, et al. Antimicrobial resistance in recent fecal enterococci from healthy volunteers and food handlers in Spain: genes and phenotypes. Microb Drug Resist. 2003;9(1):47-59.

Manuscrito recibido el 9 de abril de 2011. Aceptado para publicación, tras revisión, el 14 de septiembre de 2011.

ABSTRACT Objective. To identify infection-causing Enterococcus species in Cuban hospitals and determine their susceptibility to antimicrobial drugs, as well as their resistance mechanisms.

\section{Antimicrobial susceptibility and genetic bases for resistance of infection- causing Enterococcus strains in Cuba}

Methods. A total of 687 Enterococcus isolates from 30 Cuban hospitals in nine provinces of the country were studied over the period 2000-2009. The species were identified using both the conventional method and the automatic API ${ }^{\circledR}$ system. The minimum inhibitory concentration was determined for 13 antimicrobial drugs following the standards recommended by the Clinical Laboratory and Standards Institute. The polymerase chain reaction technique was used to characterize the genes that were resistant to aminoglycosides, erythromycin, tetracycline, and glucopeptides. The presence of beta-lactamase was determined by the chromogenic cephalosporin test.

Results. The most prevalent species were Enterococcus faecalis $(82.9 \%)$ and E. faecium (12.2\%). Resistance to glucopeptides $(1.0 \%)$ was mediated by the vanA and vanB genes. The strains resistant to ampicillin $(6 \%)$ did not produce beta-lactamases. A high percentage of resistance to aminoglycosides was observed. Gentamicin $(31.0 \%)$ and streptomycin and amikacin $(29.1 \%)$ were mediated by the aac $\left(6^{\prime}\right) I e-a p h\left(2^{\prime \prime}\right) I a$, aph (3')-IIIa, ant(6)Ia, and ant( $\left.3^{\prime \prime}\right)(9)$ genes. A correlation was found between resistance to tetracycline $(56.0 \%)$ and presence of the tet $(\mathrm{M})(75.1 \%)$ and $\operatorname{tet}(\mathrm{L})$ genes $(7.0 \%)$, while resistance to erythromycin (34.1\%) was due to the erm $(\mathrm{B})$ gene $(70.9 \%)$.

Conclusions. Resistance to vancomycin is infrequent in Cuba, as opposed to a high level of resistance to aminoglycosides, which may be indicative of treatment failures. The microbiology laboratory is a cornerstone of Enterococcus infection surveillance, along with ongoing monitoring of the susceptibility of these infections to antimicrobial drugs at a time when resistance of this microorganism is on the rise.

Key words Enterococcus; drug resistance, bacterial; Cuba. 